DOI 10.4171/JEMS/318

Qiuyi Dai · Neil S. Trudinger · Xu-Jia Wang

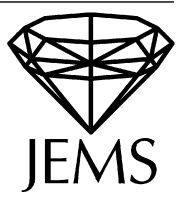

\title{
The mean curvature measure
}

Received November 26, 2007 and in revised form April 18, 2011

\begin{abstract}
We assign a measure to an upper semicontinuous function which is subharmonic with respect to the mean curvature operator, so that it agrees with the mean curvature of its graph when the function is smooth. We prove that the measure is weakly continuous with respect to almost everywhere convergence. We also establish a sharp Harnack inequality for the minimal surface equation, which is crucial for our proof of the weak continuity. As an application we prove the existence of weak solutions to the corresponding Dirichlet problem when the inhomogeneous term is a measure.
\end{abstract}

Keywords. Mean curvature measure, Harnack inequality, weak continuity of mean curvature operator, weak solution

\section{Introduction}

Notions of curvature measures arise in convex geometry (see for example [22]), and were extended to general surfaces by Federer [4] under a hypothesis of positive reach. For graphs of functions, this condition is equivalent to semiconvexity and implies twice almost everywhere differentiability by virtue of the well-known theorem of Aleksandrov. The development of a corresponding theory of curvature measures on more general sets is an open problem. Without any assumption such a theory seems impossible as the second derivative of a nonsmooth function is usually a distribution but not a measure. In this paper we consider the mean curvature and restrict ourselves to graphs of functions defined over domains $\Omega$ in Euclidean $n$-space, $\mathbb{R}^{n}$. The mean curvature has been the most extensively studied geometric quantity but usually it is regarded as a distribution when the function is not twice differentiable, such as in the case when its graph is a rectifiable set.

In particular in this paper we assign a measure to an upper semicontinuous function which is subharmonic with respect to the mean curvature operator, so that it agrees with the mean curvature of its graph when the function is smooth. We prove that the measure

Q. Dai: Department of Mathematics, Hunan Normal University, Changsha, 410081, P.R. China; e-mail: daiqiuyi@yahoo.com.cn

N. S. Trudinger, X.-J. Wang: Centre for Mathematics and its Applications,

Australian National University, Canberra ACT 0200, Australia;

e-mail: neil.trudinger@anu.edu.au,xu-jia.wang@anu.edu.au

Mathematics Subject Classification (2010): Primary 35J60; Secondary 35D05, 35R06 
is weakly continuous with respect to almost everywhere convergence (Theorem 6.1). We also establish a sharp Harnack inequality for the minimal surface equation (Theorem 2.1), which is crucial for our proof of weak continuity. As an application we prove the existence of weak solutions to the Dirichlet problem of the mean curvature equation when the right hand side is a measure (Theorem 7.1).

We say an upper semicontinuous function $u: \Omega \rightarrow[-\infty,+\infty)$ is subharmonic with respect to the mean curvature operator $H_{1}$, or $H_{1}$-subharmonic for short, if the set $\{u=-\infty\}$ has measure zero and for any open set $\omega \Subset \Omega$ and any smooth function $h \in C^{2}(\bar{\omega})$ with $H_{1}[h] \leq 0, h \geq u$ on $\partial \omega$, one has $h \geq u$ in $\omega$. As for the case of Hessian operators in $[27,28]$ this is equivalent to the inequality $H_{1}[u] \geq 0$ holding in the viscosity sense. We say a function $u$ is $H_{1}$-harmonic if it is $H_{1}$-subharmonic and for any open set $\omega \Subset \Omega$ and any $H_{1}$-subharmonic function $h$ in $\omega$ with $h \leq u$ on $\partial \omega$, one has $h \leq u$ in $\omega$. This definition does not imply directly that an $H_{1}$-harmonic function is bounded from below, but we will prove in Section 4 it is the case, and so the function is smooth. We denote the set of all $H_{1}$-subharmonic functions in $\Omega$ by $S H_{1}(\Omega)$.

A main result of the paper is the weak continuity of the mean curvature operator. That is, if $\left\{u_{k}\right\}$ is a sequence of smooth $H_{1}$-subharmonic functions which converges a.e. to $u \in S H_{1}(\Omega)$, then $H_{1}\left[u_{k}\right]$ converges weakly to the density of a measure $\mu$. The measure $\mu$ depends only on $u$ but not on the sequence $\left\{u_{k}\right\}$, so that we can assign a measure, called the mean curvature measure and denoted by $\mu_{1}[u]$, to the function $u$. Note that our measure $\mu_{1}$ is defined on $\Omega$ but Federer's measure $v_{1}$ is defined on the graph of $u$.

A crucial ingredient for the proof of the weak continuity is a refined Harnack inequality, also established in this paper, for the minimal surface equation

$$
H_{1}[u]=: \operatorname{div}\left(\frac{D u}{\sqrt{1+|D u|^{2}}}\right)=0 .
$$

Namely

$$
\sup _{B_{r}} u \leq C \inf _{B_{r}} u
$$

for nonnegative solutions of (1.1) in $\bar{B}_{2 r}$. The Harnack inequality for the mean curvature equation has been studied in several works(see for example $[3,16,19,25])$. We prove that the constant $C$ depends on the decay rate of $\left|\left\{x \in B_{2 r}: u(x)>t\right\}\right|_{n}$ (or $\mid\left\{x \in \partial B_{2 r}\right.$ : $\left.u(x)>t\}\left.\right|_{n-1}\right)$ as $t \rightarrow \infty$, where $|\cdot|_{k}$ denotes the $k$-dimensional Hausdorff measure. This is indeed the best possibility one can expect. A similar Harnack inequality also holds for the nonhomogeneous equation (see Remark 2.2).

As an application, we study the existence of solutions to the Dirichlet problem of the mean curvature equation

$$
\begin{aligned}
H_{1}[u]=v & \text { in } \Omega, \\
u=\varphi & \text { on } \partial \Omega,
\end{aligned}
$$

where $v$ is the density of a nonnegative measure with respect to Lebesgue measure.

For the Dirichlet problem of the mean curvature equation, it is usually assumed that the right hand side $v$ is a Lipschitz function, so that the interior gradient estimate holds 
and the solution is smooth, in $C^{2, \alpha}(\Omega)$ for $\alpha \in(0,1)$ [6]. If $v$ is not Lipschitz continuous, the solution may not be $C^{2}$ smooth even if $v$ is Hölder continuous (see the example in $\S 8$ ). In $[7,10]$ it was proved that when $v$ is a measurable function satisfying a necessary condition, equation (1.3) has a weak solution which is a minimizer of an associated functional. Through the mean curvature measure introduced above, we introduce a notion of weak solution and prove its existence when $v$ is a nonnegative measure.

This paper is arranged as follows. In Section 2 we establish the Harnack inequality (1.2) for the minimal surface equation. In Section 3 we establish an integral gradient estimate and a uniform estimate for $H_{1}$-subharmonic functions. In these two sections we assume that the functions are smooth. But the assumption can be removed by an approximation result proved in Section 5.

In Section 4 we introduce the Perron lifting and prove some basic properties for $\mathrm{H}_{1}$ subharmonic functions. In Section 5 we prove that every $H_{1}$-subharmonic function can be approximated by a sequence of smooth, $H_{1}$-subharmonic functions. Section 6 is devoted to the proof of the weak continuity of the mean curvature operator. The Dirichlet problem is discussed in Section 7. The final Section 8 contains some remarks.

In recent years it was proved that for several important homogeneous elliptic operators, such as the $p$-Laplace operator and the $k$-Hessian operator, one can assign a measure to a function which is subharmonic with respect to the operators, and as applications various potential-theoretical results have been established. See [12, 15, 27-30]. Our treatment of the weak continuity of the mean curvature operator was inspired by the earlier works [27-30]. However as the mean curvature operator is nonhomogeneous, the situation is much more delicate.

\section{The Harnack inequality}

In this section we prove a Harnack inequality for the minimal surface equation, which will be used for the Perron lifting process in Section 4 and the study of the Dirichlet problem in Section 7.

First we quote the basic existence and regularity result for the mean curvature equation [6]. The regularity of the mean curvature equation is based on the BombieriDe Giorgi-Miranda interior gradient estimate (see Theorem 16.5 in [6]).

Lemma 2.1. Let $u \leq 0$ be a $C^{2}$ solution to the mean curvature equation

$$
H_{1}[u]=f(x) \text { in } B_{r}(0) .
$$

Then

$$
|D u(0)| \leq C_{1} e^{C_{2}|u(0)| / r},
$$

where $C_{1}, C_{2}$ depend only on $n, r$ and $\|f\|_{C^{0,1}}$.

In Lemma 2.1, the norm $\|f\|_{C^{0,1}}$ is equal to $\sup _{B_{r}(0)}|f|$ plus the Lipschitz constant of $f$. Note that simpler proofs of the interior gradient estimate, with $|u(0)| / r$ replaced by $|u(0)|^{2} / r^{2}$, were given in $[13,31]$. These proofs also apply to the $k$-th mean curvature equation and more general Weingarten curvature equations [14, 31]. 
From the gradient estimate, the mean curvature equation becomes uniformly elliptic and one has local uniform estimates in $C^{2, \alpha}$ for any $\alpha \in(0,1)$.

The existence of classical solutions to the Dirichlet problem follows (see Theorem 16.8 in [6]).

Lemma 2.2. Let $\Omega$ be a bounded smooth domain in $\mathbb{R}^{n}$. Suppose the mean curvature of $\partial \Omega$ is nonnegative. Then for any continuous function $\varphi$ on $\partial \Omega$, there is a unique solution $u \in C^{2}(\Omega) \cap C^{0}(\bar{\Omega})$ to $H_{1}[u]=0$ such that $u=\varphi$ on $\partial \Omega$.

Lemma 2.2 also holds for the inhomogeneous equation $H_{1}[u]=f$ with $f \in C^{0,1}$, under certain conditions on $f$ and $\partial \Omega$ (see Theorem 16.10 in [6]).

In this section we prove the following Harnack inequality. Here we consider smooth solutions only. In Section 4 we will show that an $H_{1}$-harmonic function must be smooth.

Theorem 2.1. Let $u \geq 0$ be a $C^{2}$ solution to the minimal surface equation

$$
H_{1}[u]=0 \quad \text { in } B_{r}(0) .
$$

Let $\psi_{u}$ denote the distribution function of $u$, that is,

$$
\psi_{u}(t)=\left|\left\{x \in B_{r}(0): u(x)>t\right\}\right|
$$

where $|\cdot|$ denotes $n$-dimensional Lebesgue measure. Suppose there exists a positive function $\psi$ with $\psi(t) \rightarrow 0$ as $t \rightarrow \infty$ such that $\psi_{u} \leq \psi$. Then there exists a constant $C>0$ depending only on $n, r$, and $\psi$ such that

$$
\sup _{B_{r / 2}(0)} u \leq C \inf _{B_{r / 2}(0)} u .
$$

Remark 2.1. (i) The Harnack inequality (2.4) with constant $C$ depending on $\sup u$ follows immediately from the interior gradient bound and Moser's Harnack inequality [6]. This dependence can be reduced to $\int_{B_{r}} u^{p}$ for any $p>0$, by virtue of [23]. An alternative approach is given in [25], which avoids the interior gradient bound and also extends to elliptic equations satisfying certain structure conditions. Our refined estimate also extends to the equations treated in [25]. Note that by [24], (2.4) automatically holds for weak solutions, that is, for $W_{\text {loc }}^{1,1}$ solutions.

We also refer the reader to $[3,16,19]$ for further discussion of the Harnack inequality.

(ii) Recall that in the Harnack inequality for the Laplace equation, the constant $C$ depends only on $n$. But this is impossible for the minimal surface equation. One can construct a positive solution of (2.3) in $B_{1}(0)$ such that $u(0) \leq 1$ but $\int_{B_{1}} u^{p}$ can be as large as we want, for any $p>0$. To see this, let $\varphi\left(x_{1}\right)$ be a positive, convex function defined for $x_{1} \in(-1,1)$ such that $\varphi\left(x_{1}\right)$ is small when $x_{1}<1 / 4$ and $\varphi\left(x_{1}\right) \rightarrow \infty$ as $x_{1} \rightarrow 1$. Let $u$ be the solution of (2.3) with the Dirichlet condition $u=\varphi$ on $\partial B_{1}$. Then by the convexity of $\varphi, H_{1}[\varphi] \geq 0$. Hence by the comparison principle, we have $u \geq \varphi$ in $B_{1}$. Hence $\int_{B_{1}} u^{p}$ can be arbitrarily large provided $\varphi$ is sufficiently large near $x_{1}=1$. On the other hand, by constructing a suitable upper barrier one has $u(0) \leq 1$.

Theorem 2.1 follows from the following subsolution estimate, which extends the corresponding result in [23]. For convenience we will formulate and prove it for smooth functions but the proof also carries over to $W_{\text {loc }}^{1,1}$ subsolutions. 
Lemma 2.3. Let $u$ be a smooth $H_{1}$-subharmonic function in $B_{r}(0)$. Then there exists a constant $C>0$ depending only on $n, r$, and $\psi$ such that

$$
\sup _{B_{r / 2}(0)} u \leq C .
$$

Proof. It is convenient to prove Lemma 2.3 for general quasilinear divergence structure operators of the form

$$
Q u=\operatorname{div} A(\cdot, u, D u)+B(\cdot, u, D u),
$$

where $A$ maps $\Omega \times \mathbb{R} \times \mathbb{R}^{n}$ into $\mathbb{R}^{n}$ and $B$ is a scalar function on $\Omega \times \mathbb{R} \times \mathbb{R}^{n}$, satisfying a "one" structure (see [23]),

$$
|A(x, z, p)| \leq a_{0}, \quad p \cdot A(x, z, p) \geq|p|-a_{1}, \quad B \leq b_{0},
$$

for all $(x, z, p) \in \Omega \times \mathbb{R} \times \mathbb{R}^{n}$, where $a_{0}, a_{1}, b_{0} \geq 0$ are constants. The functions $A$ and $B$ are assumed to be at least measurable in the $x$ variables and continuous in $(z, p)$. The mean curvature operator $H_{1}$ clearly satisfies (2.7) with $a_{0}=a_{1}=1$ and $b_{0}=0$.

Now let $u \in C^{2}\left(B_{r}\right)$ satisfy $Q u \geq 0$ and fix $\psi$ in accordance with Theorem 2.1. Taking $r=1$, we construct from $\psi$ a function $\Phi \in C^{0,1}[0, \infty)$ such that $\Phi(0) \geq 1$, $\Phi(\infty)=\infty, 0 \leq \Phi^{\prime} \leq 1$ and

$$
\int_{B_{1}} \Phi\left(u^{+}\right)<\infty
$$

We now choose test functions

$$
v=\eta\left[\Phi\left(u^{+}\right)\right]^{\beta}
$$

in the integral form

$$
\int A \cdot D v \leq \int B v
$$

where $\eta \in C_{0}^{1}\left(B_{1}\right)$ is a cut-off function satisfying $0 \leq \eta \leq 1, u^{+}=\sup \{u, 0\}$ and $\beta \geq 1$. From the structure conditions (2.7), we thus obtain

$$
\int \eta|D \Phi|^{\beta} \leq\left(a_{0} \sup |D \eta|+a_{1} \beta+b_{0}\right) \int_{\{\eta>0\}} \Phi^{\beta} .
$$

The result then follows by Moser iteration as in [23]. For completeness we describe the argument. Applying the Sobolev inequality in (2.11), we obtain

$$
\left\|\eta \Phi^{\beta}\right\|_{n /(n-1), B_{1}} \leq C(\beta+\sup |D \eta|)\left\|\Phi^{\beta}\right\|_{1,\{\eta>0\}} .
$$

Letting $r_{v}=\left(1+2^{-v}\right) / 2, v=1,2, \ldots$, choosing $\eta=1$ on $B_{r_{v+1}}, \eta=0$ outside $B_{r_{v}}$, with $|D \eta| \leq 2^{v+2}$ and taking the $\beta$-th root, we obtain

$$
\|\Phi\|_{\beta \chi, B_{r_{v+1}}} \leq[C(\beta+\sup |D \eta|)]^{1 / \beta}\|\Phi\|_{\beta, B_{r_{v}}},
$$

where $\chi=n /(n-1)$, so that if $\beta=\chi^{v}$,

$$
\|\Phi\|_{\chi^{\nu+1}, B_{r_{v+1}}} \leq C^{\nu \chi^{-v}}\|\Phi\|_{\chi^{v}, B_{r_{v}}} .
$$


Hence as $v$ tends to $\infty$, we obtain

$$
\sup _{B_{1 / 2}} \Phi \leq C \int_{B_{1}} \Phi .
$$

The desired bound (2.5) now follows by replacing $x$ and $u$ respectively by $x / r$ and $u / r$.

By approximation in $\$ 5$, Lemma 2.3 holds for general subharmonic functions. Note that by taking $\Phi(t)=(1+t)^{p}$ in the proof of Lemma 2.3, we obtain (as in [23]) a bound for $\sup u$ in terms of $\int\left(u^{+}\right)^{p}$ for any $0<p \leq 1$, namely

$$
\sup _{B_{r / 2}} u \leq C\left[\left(r^{-n} \int_{B_{r}}\left(u^{+}\right)^{p}\right)^{1 / p}+r\right]
$$

where $C=C(n, p)$. Using [25], this can be improved to a homogeneous local maximum principle.

Corollary 2.1. Let $u$ be an $H_{1}$-subharmonic function in $B_{r}(0)$. Then for any constant $p>0$, there exists a constant $C$ depending on $n$ and $p$ such that

$$
\sup _{B_{r / 2}} u \leq \frac{C}{r^{n / p}}\left(\int_{B_{r}}\left(u^{+}\right)^{p}\right)^{1 / p},
$$

where $u^{+}=\max (u, 0)$.

Proof. From [25], (2.14) is valid with a constant $C$ depending also on $\sup _{B_{r}} u / r$. But from (2.13), we see that (2.14) holds if $r \leq \sup _{B_{r / 2}} u / 2 C$. Hence replacing $r$ by $r / 2$ we obtain the estimate (2.14) for $\sup _{B_{r / 4}} u$, from which (2.14) follows as stated.

Proof of Theorem 2.1. Let $u$ be a nonnegative solution to the minimal surface equation (2.3) in $B_{r}(0)$. From Lemma 2.3, $\sup _{B_{r / 2}(0)} u$ is bounded from above by a constant $C$ depending only on $n, r$ and $\psi$. Once $u$ is bounded from above, by the interior gradient estimate, equation (2.3) becomes uniformly elliptic and the full Harnack inequality follows [6]. Alternatively we may also use the estimates for $\inf _{B_{1 / 2}(0)} u$ in [25] or [20].

Remark 2.2. (i) By slightly modifying the proof of Lemma 2.3 , we can assume that $a_{0}, a_{1} \in L^{q}, q>n, b_{0} \in L^{n}$ in the structure conditions (2.7). From Theorem 3.1 in [25], we then obtain an extension of the Harnack inequality (2.4) to nonnegative solutions $u \in W^{2, n}(\Omega)$ of the nonhomogeneous mean curvature equation (2.1),

$$
\sup _{B_{r / 2}(0)} u \leq C\left\{\inf _{B_{r / 2}(0)} u+\|f\|_{L^{n}\left(B_{r}(0)\right)}\right\}
$$

with the constant $C$ depending on $n$ and $f$. 
(ii) In the original version of this paper (see for example [2]), we had a more complicated proof of Lemma 2.3, which in some respects was also sharper. In particular we obtained a strengthening of the above remark, namely if for any $\omega \subset \Omega$,

$$
\int_{\omega} H_{1}[u] \geq-v(\omega)
$$

for some nonnegative measure $v$ satisfying $v(\omega) /|\partial \omega| \rightarrow 0$ as $|\omega| \rightarrow 0$, then estimate (2.5) holds, with the constant $C$ depending also on $v$.

\section{Gradient and uniform estimates}

First we establish an integral gradient estimate.

Lemma 3.1. Let $u \in C^{2}(\Omega)$ be a nonpositive $H_{1}$-subharmonic function. Then for any open set $\omega \Subset \Omega$ and any $t \geq 0$,

$$
\int_{\omega}\left|D u_{t}\right| \leq C
$$

where $u_{t}=\max (u,-t), t$ is a constant, and $C>0$ depends on $\omega, t$, but is independent of $u$.

Proof. Let $\varphi \in C_{0}^{\infty}(\Omega)$ be a smooth function with compact support in $\Omega$ such that $0 \leq$ $\varphi(x) \leq 1$ and $\varphi(x) \equiv 1$ on $\omega$. We may assume that $|\partial \Omega|$, the area of $\partial \Omega$, is bounded, otherwise we may restrict to a subdomain of $\Omega$ which contains $\omega$. Then

$$
\begin{aligned}
\int_{\Omega} \varphi\left(-u_{t}\right) H_{1}[u] & =\int_{\Omega} \frac{\varphi\left|D u_{t}\right|^{2}}{\sqrt{1+\left|D u_{t}\right|^{2}}}+\int_{\Omega} \frac{u_{t} D u_{t} \cdot D \varphi}{\sqrt{1+\left|D u_{t}\right|^{2}}} \\
& \geq \int_{\omega} \frac{\left|D u_{t}\right|^{2}}{\sqrt{1+\left|D u_{t}\right|^{2}}}+\int_{\Omega} \frac{u_{t} D u_{t} \cdot D \varphi}{\sqrt{1+\left|D u_{t}\right|^{2}}} \\
& \geq \int_{\omega}\left|D u_{t}\right|-|\omega|+\int_{\Omega} \frac{u_{t} D u_{t} \cdot D \varphi}{\sqrt{1+\left|D u_{t}\right|^{2}}}
\end{aligned}
$$

where we have used the inequality $t^{2} / \sqrt{1+t^{2}} \geq t-1$. Note that

$$
\int_{\Omega} \varphi\left(-u_{t}\right) H_{1}[u] \leq t \int_{\Omega} H_{1}[u] \leq t|\partial \Omega|
$$

and

We obtain

$$
\int_{\Omega} \frac{u_{t} D u_{t} \cdot D \varphi}{\sqrt{1+\left|D u_{t}\right|^{2}}} \geq-C t|\Omega| .
$$

Hence (3.1) is proved.

$$
\int_{\omega}\left|D u_{t}\right| \leq C(1+t)(|\Omega|+|\partial \Omega|) .
$$

In the next section we will prove that every $H_{1}$-subharmonic function can be approximated by smooth ones. Note that if $u \in S H_{1}(\Omega)$, then $u_{t} \in S H_{1}(\Omega)$. Hence by Theorem 3.1 we have 
Corollary 3.1. For any $u \in S H_{1}(\Omega)$ bounded from above and any $t \in \mathbb{R}$ and $\Omega^{\prime} \Subset \Omega$, we have $u_{t} \in B V\left(\Omega^{\prime}\right)$. In particular if $u$ is bounded from below, then $u \in B V\left(\Omega^{\prime}\right)$.

By the example in $\S 8, u \notin W^{1,1}\left(\Omega^{\prime}\right)$ in general.

Next we consider the $L^{\infty}$ estimate for $H_{1}$-subharmonic functions [8]. We say a set $A$ is Caccioppoli if it is a Borel set with characteristic function $\varphi_{A}$ whose distributional derivatives $D \varphi_{A}$ are Radon measures [11]. If $A$ is Caccioppoli, we denote

$$
P_{A}=\int_{\mathbb{R}^{n}}\left|D \varphi_{A}\right|
$$

the perimeter of $A$.

Theorem 3.1. Assume that $u \in S H_{1}(\Omega) \cap C^{2}(\Omega)$ is bounded from below on $\partial \Omega$. Assume that there is a positive constant $\eta$ such that for any Caccioppoli set $A \subset \Omega$,

$$
\int_{A} H_{1}[u] \leq(1-\eta) P_{A}
$$

Then there is a constant $C>0$, depending only on $n, \Omega, \eta$, and $\inf _{\partial \Omega} u$, such that

$$
\inf _{x \in \Omega} u \geq-C .
$$

Proof. For any $t>0$, denote $\Omega_{t}=\{x \in \Omega: u(x) \leq-t\}$ and $\partial_{1} \Omega_{t}=\left\{x \in \partial \Omega_{t}\right.$ : $\left.|D u| \leq t^{2 / 3}\right\}$. Since $u$ is bounded from below on $\partial \Omega$, we may choose a large $T$ such that $\Omega_{T} \Subset \Omega$ and

$$
\frac{T^{2 / 3}}{\sqrt{1+T^{4 / 3}}} \geq 1-\eta / 2 .
$$

We claim that for a.e. $t>T$ (such that $\partial \Omega_{t}$ is smooth),

$$
\left|\partial_{1} \Omega_{t}\right| \geq \frac{\eta}{2}\left|\partial \Omega_{t}\right| \text {. }
$$

Indeed, if there exists a $t \geq T$ such that $\left|\partial_{1} \Omega_{t}\right|<\frac{\eta}{2}\left|\partial \Omega_{t}\right|$, we have

$$
\begin{aligned}
\int_{\Omega_{t}} H_{1}[u] & =\int_{\partial \Omega_{t}} \frac{|D u|}{\sqrt{1+|D u|^{2}}} \geq \int_{\partial \Omega_{t}-\partial_{1} \Omega_{t}} \frac{|D u|}{\sqrt{1+|D u|^{2}}} \\
& \geq(1-\eta / 2)(1-\eta / 2)\left|\partial \Omega_{t}\right|>(1-\eta)\left|\partial \Omega_{t}\right|,
\end{aligned}
$$

which is in contradiction with the assumption (3.3).

Let $\varphi(t)=\left|\Omega_{t}\right|$. If $t>-\inf _{\partial \Omega} u$, then $\Omega_{t} \Subset \Omega$. Hence by the co-area formula,

$$
\varphi^{\prime}(t)=-\int_{\partial \Omega_{t}} \frac{1}{|D u|} \leq-\int_{\partial_{1} \Omega_{t}} \frac{1}{|D u|} .
$$

When $t>T$,

$$
\varphi^{\prime}(t) \leq-\frac{\eta}{2 t^{2 / 3}}\left|\partial \Omega_{t}\right|
$$


By the isoperimetric inequality,

$$
\varphi^{1-1 / n}(t) \leq C\left|\partial \Omega_{t}\right|
$$

we obtain

$$
\varphi^{\prime}(t) \leq-\frac{C \eta}{t^{2 / 3}} \varphi^{1-1 / n}(t)
$$

Namely $\left[\varphi^{1 / n}(t)\right]^{\prime} \leq-C \eta t^{-2 / 3}$. Integrating from $T$ to $t$, we obtain

$$
\varphi^{1 / n}(t) \leq \varphi^{1 / n}(T)+C \eta\left(T^{1 / 3}-t^{1 / 3}\right)
$$

for a different $C$. Hence $\varphi$ vanishes when $t \geq T_{1}$, where

$$
T_{1}=C\left[T+\left(|\Omega|^{1 / n} / \eta\right)^{3}\right] .
$$

This completes the proof.

Remark 3.1. From the above proof, one sees that instead of any Caccioppoli set $A \subset \Omega$, it suffices to assume (3.3) for $\Omega_{t}$ for all $t \in\left(0, T_{1}\right]$, where $T_{1}$ is given in (3.9). Moreover, as the co-area formula holds for BV functions [11], the above argument also applies to $\mathrm{BV}$ functions.

Remark 3.2. By the approximation in Section 5, Theorem 3.1 also holds for non-smooth $H_{1}$-subharmonic functions.

Remark 3.3. There are other proofs of Theorem 3.1 (see [6]). A similar estimate for the $k$-curvature equation was established in [26]. We include a direct proof for the mean curvature case (namely, $k=1$ ) for completeness.

\section{Perron lifting}

Perron lifting for quasilinear elliptic equations has been studied in [12] and [30]. Here we adopt the treatments there to prove similar results for the mean curvature equation. Let $u$ be an $H_{1}$-subharmonic function in $\Omega$ and let $\omega \Subset \Omega$ be an open, precompact set in $\Omega$. The Perron lifting of $u$ in $\omega, u^{\omega}$, is defined as the upper semicontinuous regularization of

$$
\tilde{u}=\sup \left\{v: v \text { is } H_{1} \text {-subharmonic in } \Omega \text { and } v \leq u \text { in } \Omega-\omega\right\} \text {, }
$$

namely

$$
u^{\omega}(x)=\lim _{r \rightarrow 0} \sup _{B_{r}(x)} \tilde{u} .
$$

Remark 4.1. One may expect that $u^{\omega}=u$ on $\partial \omega$, but this may not be true. Indeed, it is obvious that $u^{\omega} \geq u$ on $\partial \omega$. However for a general open set $\omega$, it may occur that $u^{\omega}>u$ on part of the boundary $\partial \omega$, even if $u$ is a smooth function. This is easily seen by considering the Perron lifting in $\omega=B_{R}-\bar{B}_{r}$ of a radial function $u$, where $R>r$. In general one has $u^{\omega}>u$ and $u^{\omega} \neq u$ on the inner boundary $\partial B_{r}$.

First we prove the following basic result for $H_{1}$-harmonic functions. Note that our definition of $H_{1}$-harmonic functions does not imply they are bounded from below. 
Lemma 4.1. Let $u$ be an $H_{1}$-harmonic function in $\Omega$. Then $u$ is locally bounded and smooth in $\Omega$, and satisfies the equation $H_{1}[u]=0$ in $\Omega$.

Proof. Assume that $B_{1}(0) \Subset \Omega$. By definition, an $H_{1}$-harmonic function is $H_{1}$-subharmonic. The $n$-dimensional Hausdorff measure $|\{x \in \Omega: u<-t\}|_{n} \rightarrow 0$ as $t \rightarrow \infty$. Hence by a slicing and scaling argument, we may assume that the $(n-1)$-dimensional Hausdorff measure $\left|\left\{x \in \partial B_{1}: u(x)<-t\right\}\right|_{n-1} \rightarrow 0$ as $t \rightarrow \infty$.

Since $u$ is upper semicontinuous, there exists a sequence $\left\{v_{j}\right\}$ of smooth functions in $\Omega$ such that $v_{j} \searrow u$. By Lemma 2.2, there is a solution $\hat{v}_{j} \in C^{2}\left(B_{1}\right) \cap C^{0}\left(\bar{B}_{1}\right)$ to

$$
\begin{cases}H_{1}[v]=0 & \text { in } B_{1}(0), \\ v=v_{j} & \text { on } \partial B_{1} .\end{cases}
$$

Since the sequence $\hat{v}_{j}$ is decreasing and $\hat{v}_{j}>u$, it is convergent. We may assume that $\hat{v}_{j} \searrow \hat{v}$. Obviously $\hat{v} \geq u$ in $B_{1}$.

Next we show that $\hat{v} \leq u$ on $\partial B_{1}$, in the sense that for any given $x_{0} \in \partial B_{1}$,

$$
\lim _{x \rightarrow x_{0}} \hat{v}(x) \leq u\left(x_{0}\right) .
$$

As mentioned in Remark 4.1, (4.4) may not be true if the ball $B_{1}$ is replaced by an annulus $B_{R}-\bar{B}_{r}$. To prove (4.4), note that since $u$ is upper semicontinuous on $\partial B_{1}$, there is a continuous function $w$ on $\partial B_{1}$ such that $w\left(x_{0}\right)=u\left(x_{0}\right)$ and $w \geq u$ on $\partial B_{1}$. By the monotonicity of $v_{j}$ on $\partial B_{1}$, it is easy to show that for any $\varepsilon>0$, there is a $\delta>0$ such that for sufficiently large $j, v_{j}(x)<u(x)+\varepsilon$ in $\left\{x \in \partial B_{1}:\left|x-x_{0}\right| \leq \delta\right\}$. Hence by adding $C\left|x-x_{0}\right|^{2}$ to $w$ for some large $C$, we may assume that $w>v_{j}-\varepsilon$ on $\partial B_{1}$ when $j$ is sufficiently large. Let $\hat{w} \in C^{2}\left(B_{1}\right) \cap C^{0}\left(\bar{B}_{1}\right)$ be the solution of $H_{1}[\hat{w}]=0$ in $B_{1}(0)$, satisfying the boundary condition $\hat{w}=w$ on $\partial B_{1}$. Then $\hat{w} \geq \hat{v}_{j}-\varepsilon \geq \hat{v}-\varepsilon$. Since $\varepsilon>0$ is arbitrary, we obtain $u\left(x_{0}\right)=\hat{w}\left(x_{0}\right) \geq \hat{v}\left(x_{0}\right)$, and (4.4) follows from the uniform continuity of $\hat{w}$.

Extend $\hat{v}$ to the whole domain $\Omega$ so that $\hat{v}=u$ in $\Omega-B_{1}$. We claim that $\hat{v}$ is $H_{1^{-}}$ subharmonic in $\Omega$. Indeed, by (4.4) and recalling that $\hat{v} \geq u$ in $B_{1}$, we see that $\hat{v}$ is upper semicontinuous in $\Omega$ and the set $\{\hat{v}=-\infty\}$ has measure zero. Therefore by the definition in the Introduction, it suffices to show that for any open set $\omega \subset \Omega$ and any smooth function $h \in C^{2}(\bar{\omega})$ with $H_{1}[h] \leq 0$ in $\omega$ and $h \geq u$ on $\partial \omega$, one has $h \geq \hat{v}$ in $\omega$. Since $\hat{v} \geq u$ in $B_{1}$, we have $h \geq u$ on $\partial \omega$. Since $u$ is $H_{1}$-harmonic, by definition it is also $H_{1}$-subharmonic. Hence $h \geq u$ in $\omega$. Denote $\omega_{1}=\omega \cap B_{1}$. Then $H_{1}[h] \leq 0$ in $\omega_{1}$ and $h \geq u \geq \hat{v}$ on $\partial \omega_{1}$. By the comparison principle, it follows that $h \geq \hat{v}$ in $\omega_{1}$. The claim is proved.

Since $u$ is $H_{1}$-harmonic in $\Omega$ and we have just proved that $\hat{v}$ is $H_{1}$-subharmonic in $\Omega$, by definition we have $u \geq \hat{v}$ in $\Omega$. Therefore

$$
\hat{v} \equiv u \quad \text { in } B_{1} .
$$

To prove $u$ is locally uniformly bounded, it suffices to prove $\hat{v}_{j}$ is locally uniformly bounded in $B_{1}$. If $\inf _{B_{1 / 2}} \hat{v}_{j} \rightarrow-\infty$ as $j \rightarrow \infty$, by the Harnack inequality (Theorem 2.1), we see that $\hat{v}_{j} \rightarrow-\infty$ uniformly in $B_{1 / 2}$. Recall that $\hat{v}_{j} \geq u$. We obtain $u=-\infty$ in $B_{1 / 2}$. 
But by the definition of subharmonic functions, the set $\{u=-\infty\}$ has measure zero, and we reach a contradiction. Hence $\hat{v}_{j}$ is locally uniformly bounded, and so $u$ is locally uniformly bounded and smooth. Note that to apply Theorem 2.1 we need the condition $\left|\left\{u(x)<-t: x \in \partial B_{1}\right\}\right|_{n-1} \rightarrow 0$ as $t \rightarrow \infty$, which is satisfied as noted at the beginning of the proof.

Remark 4.2. The function $\hat{v}$ is independent of the sequence $v_{j}$. Indeed, let $w_{j}$ be another sequence of smooth functions on $\partial B_{1}$ such that $w_{j} \searrow u$. Let $\hat{w}_{j}$ be the solution of (4.3) with boundary condition $\hat{w}_{j}=w_{j}$ on $\partial B_{1}$ and let $\hat{w}=\lim \hat{w}_{j}$. Then by (4.4), we have $\hat{w}_{j} \geq \hat{v}$ on $\partial B_{1}$. Hence $\hat{w} \geq \hat{v}$ on $\partial B_{1}$. By the comparison principle we then have $\hat{w} \geq \hat{v}$ in $B_{1}$. Similarly we have $\hat{v} \geq \hat{w}$ in $B_{1}$. Therefore we may regard $\hat{v}$ as the solution of the Dirichlet problem $H_{1}[v]=0$ in $B_{1}$ with $v=u$ on $\partial B_{1}$.

Lemma 4.2. Let $u \in S H_{1}(\Omega)$. Then for any open set $\omega \Subset \Omega$, the Perron lifting $u^{\omega} i s$ $H_{1}$-harmonic in $\omega$ and $H_{1}$-subharmonic in $\Omega$.

Proof. The property that $u^{\omega}$ is $H_{1}$-subharmonic in $\Omega$ follows by definition. Indeed, let $E \subset \Omega$ be an open set and $h \in C^{2}(\bar{E})$ be an $H_{1}$-harmonic function satisfying $h \geq u^{\omega}$ on $\partial E$. Then for any $H_{1}$-subharmonic function $v$ in (4.1), $h \geq v$ on $\partial E$. Hence $h \geq v$ in $E$. By the definition of $u^{\omega}$ in (4.1) and (4.2) and the observation that $h \in C^{2}(\bar{E})$, it follows that $h \geq u^{\omega}$ in $E$. That is, $u^{\omega}$ is $H_{1}$-subharmonic.

To show that $u^{\omega}$ is $H_{1}$-harmonic in $\omega$, let $B_{r} \Subset \omega$ and let $v$ be the solution of the Dirichlet problem $H_{1}[v]=0$ in $B_{r}$ with $v=u^{\omega}$ on $\partial B_{r}$ (see Remark 4.2). Then $v \geq u^{\omega}$ in $B_{r}$. Let $\hat{u}=v$ in $B_{r}$ and $\hat{u}=u^{\omega}$ in $\Omega-B_{r}$. Then $\hat{u}$ is upper semicontinuous and $H_{1}$-subharmonic. It follows by (4.1) that $\hat{u} \leq u^{\omega}$. Hence $u^{\omega}=v$ in $B_{r}$. That is, $u^{\omega}$ is $H_{1}$-harmonic in $B_{r}$.

Lemma 4.3. Suppose $\left\{u_{j}\right\} \subset S H_{1}(\Omega)$ converges to a measurable function $u$ a.e. with $|\{u=-\infty\}|=0$. Let $\tilde{u}$ be the upper semicontinuous regularization of $u$. Then $\tilde{u}=u$ a.e. and $\tilde{u}$ is $H_{1}$-subharmonic.

Proof. For any constant $t>0$, denote $u_{t, j}=\max \left(u_{j},-t\right), u_{t}=\max (u,-t)$, and $\tilde{u}_{t}$ the upper semicontinuous regularization of $u_{t}$. It is easy to check that if Lemma 4.3 holds for $u_{t}$ for all $t>0$, then it also holds for $u$. Therefore in the following proof we may assume that the $u_{j}$ are uniformly bounded from below.

To prove $u=\tilde{u}$ a.e. in $\Omega$, let $x_{0}$ be a Lebesgue point of $u$ and $u_{j}$, and assume $u_{j}\left(x_{0}\right) \rightarrow u\left(x_{0}\right)$. By adding proper constants to $u_{j}$ and $u$, we assume that $u_{j}\left(x_{0}\right)=$ $u\left(x_{0}\right)=0$. Then by approximation by smooth functions in $S H_{1}(\Omega)$ (see Section 5 below), Lemma 2.3 implies that $\sup _{B_{r}\left(x_{0}\right)} u_{j} \rightarrow 0$ as $r \rightarrow 0$, uniformly in $j$. Hence $u\left(x_{0}\right)=\tilde{u}\left(x_{0}\right)$.

To prove that $\tilde{u}$ is $H_{1}$-subharmonic, let $\omega \Subset \Omega$ be an open set and $h \in C^{2}(\bar{\omega})$ be an $H_{1}$-harmonic function with $h \geq \tilde{u}$ on $\partial \omega$. If $u_{j}$ is decreasing, then for any $\varepsilon>0$, by the upper semicontinuity of $u_{j}$ and Lemma $2.3, h \geq u_{j}-\varepsilon$ on $\partial \omega$ provided $j$ is sufficiently large. It follows that $h \geq u_{j}-\varepsilon$ in $\omega$ for all large $j$. Hence $h \geq \tilde{u}$ in $\omega$ and so $\tilde{u}$ is $H_{1}$-subharmonic. If $u_{j}$ is increasing, obviously $h \geq u_{j}$ on $\partial \omega$ for all $j$. Hence $h \geq \tilde{u}$ in $\omega$ and so $\tilde{u}$ is $H_{1}$-subharmonic. 
For general $\left\{u_{j}\right\}$, let $w_{k, j}=\max \left\{u_{k}, \ldots, u_{j}\right\}$. Then for fixed $k, w_{k, j} \nearrow w_{k}$ a.e. as $j \rightarrow \infty$, for some $w_{k} \in S H_{1}(\Omega)$, and $w_{k} \searrow u$ a.e. as $k \rightarrow \infty$. Hence $u$ is $H_{1^{-}}$ subharmonic.

Suppose $B_{r}:=B_{r}\left(x_{0}\right) \subset \Omega$ for $0<r \leq r_{0}$. For $u \in S H_{1}(\Omega)$, the Perron lifting $u^{B_{t}}$ is increasing in $t$, and

$$
\lim _{t \rightarrow \delta^{-}} u^{B_{t}}(x) \leq u^{B_{\delta}}(x) \leq \lim _{t \rightarrow \delta^{+}} u^{B_{t}}(x) \quad \forall x \in \Omega, t<r_{0} .
$$

This implies that $\left\|\varphi\left(u^{B_{t}}\right)\right\|_{L^{1}(\Omega)}$, as a function of $t$, is monotone and bounded, where

$$
\varphi(t)=\arctan t
$$

We introduce the function $\varphi$ since $u$ may fail to be in $L_{\text {loc }}^{1}(\Omega)$. Hence, $\left\|\varphi\left(u^{B_{t}}\right)\right\|_{L^{1}(\Omega)}$ is continuous for almost all $t$. Since $u^{B_{t}}$ is continuous in $B_{t}$, it follows that

$$
\lim _{t \rightarrow r}\left|\varphi\left(u^{B_{t}}(x)\right)-\varphi\left(u^{B_{r}}(x)\right)\right| \rightarrow 0 \quad \text { for a.e. } r \in\left(0, r_{0}\right),
$$

Similar to Lemma 3.6 in [30], we have the following

Lemma 4.4. Suppose $u_{j}, u \in S H_{1}(\Omega)$ and $u_{j} \rightarrow u$ a.e. in $\Omega$. Then for any $r \in\left(0, r_{0}\right)$ such that (4.6) holds for $u$ and $u_{j}$, we have $u_{j}^{B_{r}} \rightarrow u^{B_{r}}$ a.e. in $\Omega$ as $j \rightarrow \infty$.

Proof. Since $u_{j}^{B_{r}}$ and $u^{B_{r}}$ are locally uniformly bounded in $C_{\mathrm{loc}}^{2}\left(B_{r}\right)$, by passing to a subsequence we may assume that $u_{j}^{B_{r}}$ is convergent. Let $w^{\prime}=\lim u_{j}^{B_{r}}$, and let $w$ be the upper semicontinuous regularization of $w^{\prime}$ (note that $w$ and $w^{\prime}$ can differ only on $\partial B_{r}$ ). Then $w \in S H_{1}(\Omega)$ and $w=u$ in $\Omega-\bar{B}_{r}$. Hence by the definition of the Perron lifting, we have $u^{B_{r}} \geq w$.

Next we prove that $w \geq u^{B_{r-\delta}}$ for any $\delta>0$. Once this is proved, we have $u^{B_{r}} \geq$ $w \geq u^{B_{r-\delta}}$. Sending $\delta \rightarrow 0$, we obtain $u^{B_{r}}=w$ by (4.6).

To prove $w \geq u^{B_{r-\delta}}$, it suffices to prove that for any $\varepsilon>0, u_{j}^{B_{r}} \geq u-\varepsilon$ on $\partial B_{r-\delta}$ for sufficiently large $j$. By the interior gradient estimate, $u_{j}^{B_{r}}$ is uniformly bounded in $C^{2}\left(B_{r-\delta / 4}\right)$. If there exists a point $y_{0} \in \partial B_{r-\delta}$ and a fixed $\varepsilon>0$ such that $u\left(y_{0}\right)>$ $u_{j}^{B_{r}}\left(y_{0}\right)+\varepsilon$ for all large $j$, then by Lemma 2.3 , there is a Lebesgue point $y_{1} \in B_{\delta / 4}\left(y_{0}\right)$ of $u$ such that $u\left(y_{1}\right)>u_{j}^{B_{r}}\left(y_{1}\right)+\frac{1}{2} \varepsilon$ for all large $j$. It follows that the limit function $w=\lim _{j \rightarrow \infty} u_{j}^{B_{r}}$ is strictly less than $u$ a.e. near $y_{1}$. We reach a contradiction as $w=$ $\lim _{j \rightarrow \infty} u_{j}^{B_{r}} \geq \lim _{j \rightarrow \infty} u_{j}=u$.

\section{Approximation by smooth functions}

Using the technique of Perron lifting, we prove that every $H_{1}$-subharmonic function can be approximated by a sequence of smooth $H_{1}$-subharmonic functions. 
Theorem 5.1. For any $u \in S H_{1}(\Omega)$, there is a sequence $\left\{u_{j}\right\} \subset S H_{1}\left(\Omega_{2^{-j}}\right)$ of smooth functions such that $u_{j} \rightarrow u$ a.e. on $\Omega$, where $\Omega_{\delta}=\{x \in \Omega: \operatorname{dist}(x, \partial \Omega)>\delta\}$.

Proof. The proof is divided into two steps. In the first one we use the Perron lifting to get a sequence $\left\{u_{j}\right\}$ of piecewise smooth $H_{1}$-subharmonic functions which converges to $u$ a.e. In the second one we modify $u_{j}$ to get a sequence of smooth $H_{1}$-subharmonic functions.

Step 1. For each $j=1,2, \ldots$, let $\left\{B_{j, k}: k=1, \ldots, k_{j}\right\}$ be a family of finitely many balls of radius $2^{-j}$, contained in $\bar{\Omega}$, such that $\Omega_{2^{-j-1}} \subset \bigcup_{k=1}^{k_{j}} B_{j, k}$.

Let $u_{j, 0}=u$. For $m=1, \ldots, k_{j}$, define $u_{j, m}$ so that $u_{j, m}=u_{j, m-1}$ in $\Omega-B_{j, m}$ and $u_{j, m}$ is the solution of

$$
\begin{cases}H_{1}[v]=0 & \text { in } B_{j, m}, \\ v=u_{j, m-1} & \text { on } \partial B_{j, m} .\end{cases}
$$

and denote $u_{j}=u_{j, k_{j}}$. Then $u_{j}$ is a sequence of piecewise smooth $H_{1}$-subharmonic functions and

$$
u_{j} \geq u \text {. }
$$

To show that $u_{j} \rightarrow u$ a.e., recall that every upper semicontinuous function $u$ can be approximated by a decreasing sequence $\left\{v^{m}\right\}$ of smooth functions, $v^{m} \searrow u$ a.e. For each $m$, define $v_{j}^{m}$ as above. Then $v_{j}^{m} \rightarrow v^{m}$ as $j \rightarrow \infty$. Hence we may choose $j=j_{m}$ so large that $v_{j_{m}}^{m} \rightarrow u$ a.e. Note that $v_{j_{m}}^{m} \geq u_{j_{m}}$. Hence $u_{j} \rightarrow u$ a.e.

Step 2. The functions $u_{j}$ are piecewise smooth. To get a smooth sequence we modify $u_{j, k}$ as follows. For a fixed $j$, let $u_{j, 1}$ and $u_{j, 2}$ be as in Step 1 . The function $u_{j, 2}$ is piecewise smooth in $B_{j, 1} \cup B_{j, 2}$, its gradient may have a jump across $\Gamma:=B_{j, 1} \cap \partial B_{j, 2}$. If $D u_{j, 2}$ has a jump at some point on $\Gamma$, then by the maximum principle, we have $u_{j, 2}>u_{j, 1}$ in $B_{j, 2}-B_{j, 1}$. By the Hopf lemma, $D u_{j, 2}$ has a jump at every point on $\Gamma$.

We modify $u_{j, 2}$ near $\Gamma$ as follows. First we make a coordinate transform such that

$$
\Gamma=\left\{x_{n}=0\right\} \times\left\{\left|x^{\prime}\right|<r\right\} .
$$

Let

$$
a\left(x^{\prime}\right)=\partial_{x_{n}} u_{j, 2}\left(x^{\prime}, 0\right)-\partial_{x_{n}} u_{j, 1}\left(x^{\prime}, 0\right) .
$$

By the Hopf lemma, $a\left(x^{\prime}\right)>0$ for all $\left|x^{\prime}\right|<r$. Let

$$
\varphi(x)=\frac{a\left(x^{\prime}\right)}{4 \varepsilon \rho\left(x^{\prime}\right)}\left(x_{n}+\varepsilon \rho\left(x^{\prime}\right)\right)^{2},
$$

where $\varepsilon \ll 2^{-j}$ is a constant and $B_{r}^{\prime}(0)$ is a ball in $\mathbb{R}^{n-1}$. We choose $\rho \in C^{\infty}\left(B_{r}^{\prime}(0)\right)$ satisfying $0<\rho\left(x^{\prime}\right)<a\left(x^{\prime}\right)$ in $B_{r}^{\prime}(0)$ and $\rho=0$ on $\partial B_{r}^{\prime}(0)$. Now let

$$
\tilde{u}_{j, 2}(x)= \begin{cases}u_{j, 2}(x) & \text { if }\left|x_{n}\right| \geq \varepsilon \rho\left(x^{\prime}\right), \\ u_{j, 2}(x)+\varphi(x) & \text { if }-\varepsilon \rho\left(x^{\prime}\right) \leq x_{n} \leq 0, \\ u_{j, 2}(x)+\varphi(x)-a\left(x^{\prime}\right) x_{n} & \text { if } 0 \leq x_{n} \leq \varepsilon \rho\left(x^{\prime}\right)\end{cases}
$$

It is obvious that $\tilde{u}_{j, 2} \in C^{1,1}$ and

$$
D_{x_{n}}^{2} \varphi(x) \geq \varepsilon^{-1} / 2 \quad \text { if }-\varepsilon \rho\left(x^{\prime}\right)<x_{n}<\varepsilon \rho\left(x^{\prime}\right),
$$


where $O(1)$ means a quantity bounded by a constant independent of $\varepsilon$. Hence $\partial_{x_{n}}^{2} \tilde{u}_{j, 2} \geq$ $1 / 2 \varepsilon-C$ is dominating in the mean curvature

$$
\left(\delta_{i j}-\frac{w_{i} w_{j}}{1+|D w|^{2}}\right) w_{i j}=\frac{H}{\sqrt{1+|D w|^{2}}} .
$$

Changing back to the original coordinates, we see that $\tilde{u}_{j, 2}$ is $H_{1}$-subharmonic when $\varepsilon$ is sufficiently small.

After the modification, $u_{j, 2}$ is smooth in $B_{j, 1} \cup B_{j, 2}$. Next we go back to Step 1 to get the function $u_{j, 3}$, which is the solution of (5.1) with $m=3$. We then repeat the above modification for $u_{j, 3}$ with a smaller $\varepsilon$. By this process we obtain a sequence of $C^{1,1}$ smooth $H_{1}$-subharmonic functions which converges to $u$.

Remark 5.1. By choosing the function $\varphi$ in (5.5) more carefully, we can make the function $\tilde{u}_{j, 2}$ in (5.6) $C^{2,1}$ smooth, or $C^{k, 1}$ smooth for any $k \geq 2$. Hence the above argument can give a sequence of smooth $H_{1}$-subharmonic functions converging to $u$.

If $\partial \Omega$ has positive mean curvature (this condition is satisfied throughout this paper when the approximation is used), we can also get a smooth sequence from the $C^{1,1}$ smooth one as follows. If $u \in C^{1,1}(\Omega)$ is $H_{1}$-subharmonic, then $H_{1}[u]=f$ for some nonnegative $f \in L^{\infty}(\Omega)$. Choose $f_{\sigma} \geq 0, f_{\sigma}=0$ at $\partial \Omega$, and $f_{\sigma} \rightarrow f$ in $L^{n}(\Omega)$. Let $u_{\sigma}$ be the solution of $H_{1}\left[u_{\sigma}\right]=f_{\sigma}$ in $\Omega$ such that $u_{\sigma}=u$ on $\partial \Omega$. Then $u_{\sigma} \rightarrow u$ by Aleksandrov's maximum principle.

Remark 5.2. We may also construct a suitable approximating sequence by using supconvolution and the fact that an $H_{1}$-subharmonic function is a viscosity subsolution. There are some subtle technicalities involved here as we must first truncate from below and subsequently mollify the sup-convolution which is only semiconvex. We thus obtain a smooth sequence $u_{m}$ converging to $u$ almost everywhere and satisfying

$$
H_{1}\left[u_{k}\right] \geq \delta_{k},
$$

where $\delta_{k} \rightarrow 0$, boundedly. This suffices to extend Lemma 2.3 to general subharmonic functions, taking account of Remark 2.2. But by invoking the existence theory again similarly to Remark 5.1, we can deduce Theorem 5.1 at least for domains with positive mean curvature as above.

\section{Weak convergence}

For $u \in S H_{1} \cap C^{2}$, denote by $\mu_{1}[u]=H_{1}[u] d x$ the associated measure. In this section, we prove the following weak convergence result for $H_{1}[u]$.

Lemma 6.1. Let $u_{j} \in C^{2}(\Omega)$ be a sequence of $H_{1}$-subharmonic functions which converges to $u \in S H_{1}(\Omega)$ a.e. in $\Omega$. Then $\left\{\mu_{1}\left[u_{j}\right]\right\}$ converges to a measure $\mu$ weakly. 
Proof. For any open set $\omega \subset \Omega$,

$$
\mu_{1}\left[u_{j}\right](\omega) \leq \mu_{1}\left[u_{j}\right](\Omega) \leq|\partial \Omega|
$$

is uniformly bounded. Hence there is a subsequence of $\mu_{1}\left[u_{j}\right]$ which converges weakly to a measure $\mu$. We need to prove that $\mu$ is independent of the choice of subsequences of $\left\{u_{j}\right\}$. and

Let $\left\{u_{j}\right\},\left\{v_{j}\right\} \subset S H_{1}(\Omega) \cap C^{2}(\Omega)$. Suppose both sequences converge to $u$ a.e. in $\Omega$

$$
\mu_{1}\left[u_{j}\right] \rightarrow \mu, \quad \mu_{1}\left[v_{j}\right] \rightarrow v
$$

weakly as measures. We claim that for any ball $B_{r}:=B_{r}\left(x_{0}\right)$ such that $B_{2 r} \subset \Omega$,

$$
\mu\left(B_{r}\right)=v\left(B_{r}\right)
$$

or equivalently, for any $t>0$,

$$
\mu\left(B_{r}\right) \leq v\left(B_{r+t}\right), \quad v\left(B_{r}\right) \leq \mu\left(B_{r+t}\right) .
$$

To get (6.3) from (6.4), note that (6.4) implies that

$$
\mu\left(B_{r-2^{-k+1}}\right) \leq v\left(B_{r-2^{k}}\right), \quad v\left(B_{r-2^{-k+1}}\right) \leq \mu\left(B_{r-2^{k}}\right) .
$$

Sending $k \rightarrow \infty$ we get (6.3).

We choose finitely many small balls $\left\{B_{l}\right\}_{l=1}^{k}$ contained in $B_{r+4 t / 5}-B_{r+t / 5}$ such that the center of each ball is on $\partial B_{r+t / 2}$ and $\bar{B}_{r+3 t / 4}-B_{r+t / 4} \subset \bigcup_{l=1}^{k} B_{l}$. Now let $u_{j, 1}$ be the Perron lifting of $u_{j}$ on $B_{1}$, and let $u_{j, 2}$ be the Perron lifting of $u_{j, 1}$ on $B_{2}, \ldots$, and let $u_{j, k}$ be the Perron lifting of $u_{j, k-1}$ on $B_{k}$. Denote $u_{j}^{t}=u_{j, k}$. Similarly we obtain $v_{j}^{t}$ and $u^{t}$. Then $u_{j}^{t}, v_{j}^{t}$ and $u^{t}$ are piecewise smooth in $B_{r+3 t / 4}-B_{r+t / 4}$, and $u_{j}^{t}=u_{j}, v_{j}^{t}=v_{j}$ in $B_{r}$, and so are smooth in $B_{r}$. By Lemma 4.4, we have

$$
u_{j}^{t}, v_{j}^{t} \rightarrow u^{t} \quad \text { in } \Omega \text { a.e. }
$$

and

$$
D u_{j}^{t}, D v_{j}^{t} \rightarrow D u^{t} \quad \text { on } \partial B_{r+t / 2} \text { a.e. }
$$

Note that to use Lemma 4.4 , we need to choose the radii of the balls $\left\{B_{l}\right\}_{l=1}^{k}$ such that the above Perron liftings satisfy (4.6).

Let $u_{j, \varepsilon}^{t}=u_{j}^{t} * \rho_{\varepsilon}$ and $v_{j, \varepsilon}^{t}=v_{j}^{t} * \rho_{\varepsilon}$ be the mollifications of $u_{j}^{t}$ and $v_{j}^{t}$, where $\rho_{\varepsilon}=\varepsilon^{-n} \rho(x / \varepsilon)$ and $\rho \in C_{0}^{\infty}\left(B_{1}(0)\right)$ is a mollifier. Since the $u_{j}$ are smooth, by our construction of $u_{j}^{t}, \Gamma_{j}^{t}$ consists of finitely many smooth hypersurfaces contained in the union $\Gamma:=\bigcup_{i=1}^{k} \partial B_{l}$ of spheres, where $\Gamma_{j}^{t}$ denotes the set of nonsmooth points of $u_{j}^{t}$. Hence $u_{j, \varepsilon}^{t}$ converges to $u_{j}^{t}$ smoothly in $\Omega_{\varepsilon}-N_{\varepsilon}(\Gamma)$, where $\Omega_{\varepsilon}=\{x \in \Omega: \operatorname{dist}(x, \partial \Omega)>\varepsilon\}$ and $N_{\varepsilon}(\Gamma)$ denotes the $\varepsilon$-neighborhood of $\Gamma$. It follows that

$$
H_{1}\left[u_{j, \varepsilon}^{t}\right] \geq-\delta_{\varepsilon} \quad \text { in } \Omega_{\varepsilon}-N_{\varepsilon}(\Gamma),
$$


with $\delta_{\varepsilon} \rightarrow 0$ as $\varepsilon \rightarrow 0$ (for any fixed $j$ ). In the subdomain $N_{\varepsilon}(\Gamma) \cap B_{r+2 t}$, since $u_{j}$ are smooth, $u_{j}^{t}$ are semiconvex. We have

$$
H_{1}\left[u_{j, \varepsilon}^{t}\right] \geq-M_{j} \quad \text { in } N_{\varepsilon}(\Gamma) \cap B_{r+2 t}
$$

and

$$
\int_{N_{\varepsilon}(\Gamma) \cap B_{r+2 t}} H_{1}\left[u_{j, \varepsilon}^{t}\right] \leq C M_{j} \varepsilon \rightarrow 0 \quad \text { as } \varepsilon \rightarrow 0,
$$

where $M_{j}$ depends on $u_{j}$ but is independent of $\varepsilon$.

Noting that $u_{j}^{t}$ is independent of $t$ in $B_{r}$, we have

$$
\begin{aligned}
\int_{B_{r}} H_{1}\left[u_{j}\right] & =\int_{B_{r}} H_{1}\left[u_{j}^{t}\right]=\lim _{\varepsilon \rightarrow 0} \int_{B_{r}} H_{1}\left[u_{j, \varepsilon}^{t}\right] \leq \lim _{\varepsilon \rightarrow 0} \int_{B_{r+t / 2}} H_{1}\left[u_{j, \varepsilon}^{t}\right] \\
& =\lim _{\varepsilon \rightarrow 0} \int_{\partial B_{r+t / 2}} \frac{\gamma \cdot D u_{j, \varepsilon}^{t}}{\sqrt{1+\left|D u_{j, \varepsilon}^{t}\right|^{2}}}=\int_{\partial B_{r+t / 2}} \frac{\gamma \cdot D u_{j}^{t}}{\sqrt{1+\left|D u_{j}^{t}\right|^{2}}},
\end{aligned}
$$

where $\gamma$ denotes the unit outer normal. Recall that $u_{j}^{t}, v_{j}^{t}$ and $u^{t}$ are piecewise smooth in $B_{r+3 t / 4}-B_{r+t / 4}$, we have

$$
\lim _{j \rightarrow \infty} \int_{\partial B_{r+t / 2}} \frac{\gamma \cdot D u_{j}^{t}}{\sqrt{1+\left|D u_{j}^{t}\right|^{2}}}=\int_{\partial B_{r+t / 2}} \frac{\gamma \cdot D u^{t}}{\sqrt{1+\left|D u^{t}\right|^{2}}} .
$$

Similarly we have

$$
\begin{aligned}
\int_{\partial B_{r+t / 2}} \frac{\gamma \cdot D u^{t}}{\sqrt{1+\left|D u^{t}\right|^{2}}} & =\lim _{j \rightarrow \infty} \int_{\partial B_{r+t / 2}} \frac{\gamma \cdot D v_{j}^{t}}{\sqrt{1+\left|D v_{j}^{t}\right|^{2}}} \\
& =\lim _{j \rightarrow \infty} \lim _{\varepsilon \rightarrow 0} \int_{\partial B_{r+t / 2}} \frac{\gamma \cdot D v_{j, \varepsilon}^{t}}{\sqrt{1+\left|D v_{j, \varepsilon}^{t}\right|^{2}}} .
\end{aligned}
$$

Note that

$$
\int_{\partial B_{r+t}} \frac{\gamma \cdot D v_{j, \varepsilon}^{t}}{\sqrt{1+\left|D v_{j, \varepsilon}^{t}\right|^{2}}}-\int_{\partial B_{r+t / 2}} \frac{\gamma \cdot D v_{j, \varepsilon}^{t}}{\sqrt{1+\left|D v_{j, \varepsilon}^{t}\right|^{2}}}=\int_{B_{r+t}-B_{r+t / 2}} H_{1}\left[v_{j, \varepsilon}^{t}\right]
$$

and $H_{1}\left[v_{j, \varepsilon}^{t}\right] \geq-\delta_{\varepsilon}$ with $\delta_{\varepsilon} \rightarrow 0$ as $\varepsilon \rightarrow 0$. Hence the right hand side of (6.8) is

$$
\leq \lim _{j \rightarrow \infty} \lim _{\varepsilon \rightarrow 0} \int_{\partial B_{r+t}} \frac{\gamma \cdot D v_{j, \varepsilon}^{t}}{\sqrt{1+\left|D v_{j, \varepsilon}^{t}\right|^{2}}}
$$

Note that $v_{j, \varepsilon}^{t}$ is independent of $t$ on $\partial B_{r+t}$. The above equals

$$
\lim _{j \rightarrow \infty} \lim _{\varepsilon \rightarrow 0} \int_{B_{r+t}} H_{1}\left[v_{j, \varepsilon}\right]=\lim _{j \rightarrow \infty} \int_{B_{r+t}} H_{1}\left[v_{j}\right] .
$$

Hence $\mu\left(B_{r}\right) \leq v\left(B_{r+t}\right)$. Similarly, we can prove $v\left(B_{r}\right) \leq \mu\left(B_{r+t}\right)$. 
From the above lemma, we can assign a measure $\mu$ to $u$ for any $u \in S H_{1}(\Omega)$, and obtain the following weak convergence theorem.

Theorem 6.1. For any $u \in S H_{1}(\Omega)$, there exists a Radon measure $\mu_{1}[u]$ such that

(i) $\mu_{1}[u]=H_{1}[u] d x$ if $u \in C^{2}(\Omega)$,

(ii) if $\left\{u_{j}\right\} \subset S H_{1}(\Omega)$ is a sequence which converges to $u$ a.e., then $\mu_{1}\left[u_{j}\right] \rightarrow \mu_{1}[u]$ weakly as measures.

Note that in (ii) above, we need to use the approximation in Section 5.

Remark 6.1. If $\left\{u_{j}\right\}$ is a sequence of semiconvex functions converging to $u$, then the weak convergence $\mu_{1}\left[u_{j}\right] \rightarrow \mu_{1}[u]$ is a special case of the weak continuity of Federer [4].

\section{Existence of weak solutions}

In this section we consider the Dirichlet problem

$$
\begin{aligned}
& H_{1}[u]=v \quad \text { in } \Omega, \\
& u=\varphi \quad \text { on } \partial \Omega \text {, }
\end{aligned}
$$

where $\Omega$ is a bounded domain in $\mathbb{R}^{n}$ with $C^{3}$ boundary, $\varphi$ is a continuous function on $\partial \Omega$, and $v$ is a nonnegative measure. Here we also use $v$ to denote its density with respect to the Lebesgue measure.

For the Dirichlet problem of the mean curvature equation, usually one assumes that the right hand side $v$ is Lipschitz continuous so that the solution is smooth $[6,9]$. When $v \in L^{n}(\Omega)$, the existence of a generalized solution, introduced in [18], was investigated in [7-10]. Here we consider solutions in $S H_{1}(\Omega)$. We say $u \in S H_{1}(\Omega)$ is a weak solution of (1.3) if $\mu_{1}[u]=v$.

Assume that for any Caccioppoli set $\omega \subset \Omega$ with nonzero measure,

$$
v(\omega)<P_{\omega},
$$

where $P_{\omega}$ is the perimeter of $\omega$, as defined in (3.2). This is also a necessary condition for the existence of smooth solutions to the mean curvature equation (7.1), which can be verified easily by integration by parts in the equation.

Let $\varphi_{\sigma} \in C_{0}^{\infty}(\Omega)$ be a nonnegative function satisfying $\varphi=1$ in $\Omega_{2 \sigma}, \varphi_{\sigma}=0$ in $\Omega-\Omega_{\sigma}$, and $0 \leq \varphi_{\sigma} \leq 1$ in $\Omega$. Let $\rho$ be a mollifier and let $g_{\sigma, \varepsilon}(x)$ be the mollification of $\nu_{\sigma}:=\varphi_{\sigma} v$, namely

$$
g_{\sigma, \varepsilon}(x)=\int_{\Omega} \rho_{\varepsilon}(x-y) d v_{\sigma}
$$

where $0<\varepsilon<\sigma$. Then $g_{\sigma, \varepsilon} \in C^{\infty}(\Omega)$ and $g_{\sigma, \varepsilon} d x$ converges to $v_{\sigma}$ weakly.

Lemma 7.1. For any open set $\omega \subset \Omega$,

$$
\int_{\omega} g_{\sigma, \varepsilon} d x<P_{\omega}
$$


Proof. Apparently $v_{\sigma}$ satisfies (7.2) for all Caccioppoli sets $\omega \subset \mathbb{R}^{n}$. Hence

$$
\int_{\omega} g_{\sigma, \varepsilon} d x=\int_{\omega} d x \int_{\Omega} \rho_{\varepsilon}(x-y) d v_{\sigma}=\int_{|z| \leq 1} v_{\sigma}(\omega-\varepsilon z) \rho(z) d z,
$$

where $\omega-\varepsilon z=\left\{x \in \mathbb{R}^{n}: x+\varepsilon z \in \omega\right\}$. By (7.2), $v_{\sigma}(\omega-\varepsilon z)<P_{\omega}$. Hence we obtain (7.3).

Consider the approximating problem

$$
\begin{aligned}
H_{1}[u] & =g_{\sigma, \varepsilon}(x) & & \text { in } \Omega, \\
u & =\varphi & & \text { on } \partial \Omega .
\end{aligned}
$$

Lemma 7.2. Under the assumption (7.3), there is a minimizer $u_{\sigma, \varepsilon}$ of the functional

$$
\mathcal{F}(u)=\int_{\Omega} \sqrt{1+|D u|^{2}}-\int_{\Omega} g_{\sigma, \varepsilon} u+\int_{\partial \Omega}|u-\varphi|
$$

in $\mathrm{BV}(\Omega)$ for every $\varphi \in L^{1}(\partial \Omega)$. If $\partial \Omega$ is $C^{3}$ smooth and $\varphi \in C^{3}(\partial \Omega)$, and the mean curvature $H^{\prime}$ of $\partial \Omega$ (with respect to the inner normal) satisfies

$$
H^{\prime}(x)>\frac{n}{n-1} g_{\sigma, \varepsilon}(x)(=0) \quad \forall x \in \partial \Omega,
$$

then $u_{\sigma, \varepsilon}=\varphi$ on $\partial \Omega$ and $u_{\sigma, \varepsilon} \in C^{2}(\bar{\Omega})$.

For the first part of Lemma 7.2, we refer the reader to [7]. For the second part, we refer to $[9,6]$.

Theorem 7.1. Let $\Omega$ be a bounded domain in $\mathbb{R}^{n}$ with $C^{3}$ boundary. Let $v$ be a nonnegative measure which satisfies (7.2) and can be decomposed as $v=v_{1}+f$ for some nonnegative measure $v_{1}$ with compact support in $\Omega$ and some Lipschitz function $f \geq 0$. Suppose the boundary mean curvature satisfies

$$
H^{\prime}(x)>\frac{n}{n-1} f(x) \quad \forall x \in \partial \Omega
$$

Then (7.1) has a weak solution.

Proof. We will prove Theorem 7.1 for $\varphi \in C^{3}(\partial \Omega)$. If $\varphi \in C^{0}(\partial \Omega)$, we can choose a sequence of functions $\varphi_{k} \in C^{3}(\partial \Omega)$ such that $\varphi_{k} \rightarrow \varphi$ and take the limit. We divide the proof into two steps.

Step 1. First we prove the theorem under the additional assumption that there exists a positive constant $\eta>0$ such that for any Caccioppoli set $\omega \subset \Omega$,

$$
v(\omega) \leq(1-\eta) P_{\omega} .
$$


Let $g_{\sigma, \varepsilon}$ be the mollification of $v$ as above, where $\sigma>\varepsilon>0, \sigma<\operatorname{dist}(S, \partial \Omega)$, and $S=\operatorname{supp} v_{1}$. Since $f \geq 0$, (7.7) implies (7.6). Hence by Lemma 7.2, there is a smooth solution $u_{\sigma, \varepsilon}$ to (7.4). By Theorem 3.1, $u_{\sigma, \varepsilon}$ is uniformly bounded,

$$
\sup _{\partial \Omega} \varphi \geq u_{\sigma, \varepsilon} \geq-C
$$

for some $C>0$ independent of $\sigma$ and $\varepsilon$. By assumption, $v$ is given by a Lipschitz continuous function $f$ in $\Omega-S$. Hence $u_{\sigma, \varepsilon}$ is locally uniformly bounded in $C^{2}(\Omega-S) \cap$ $C^{0}(\bar{\Omega}-S)$ for $0<\varepsilon<\sigma$. By Lemma 3.1, $u_{\sigma, \varepsilon}$ is uniformly bounded in $W^{1,1}\left(\Omega^{\prime}\right)$ for any $\Omega^{\prime} \Subset \Omega$. Letting $\sigma=2 \varepsilon$ and sending $\varepsilon \rightarrow 0$, we may assume that $u_{\sigma, \varepsilon}$ converges in $L^{1}$ to a limit function $u$. Note that $g_{\sigma, \varepsilon} d x$ converges weakly to $v$. By Theorem 6.1, $u$ is a weak solution of (7.1). By Corollary 3.1 and since $v=f$ is Lipschitz continuous in $\Omega-S$, it follows that $u \in \mathrm{BV}(\Omega)$.

Step 2. By Remark 3.1, Theorem 3.1 applies in Step 1 provided (7.8) holds for some sublevel sets. In this step we prove (7.8) for these sublevel sets. For any small constant $\delta \in(0,1)$, from Step 1 there is a solution $u_{\delta} \in \mathrm{BV}(\Omega)$ to

$$
\begin{aligned}
H_{1}[u] & =(1-\delta) \nu & & \text { in } \Omega, \\
u & =\varphi & & \text { on } \partial \Omega .
\end{aligned}
$$

Note that $u_{\delta}$ is the limit of smooth solutions and the comparison principle holds for smooth solutions. It follows that $u_{\delta}$ is monotone, namely $u_{\delta_{1}} \geq u_{\delta_{2}}$ if $\delta_{1}>\delta_{2}$. Moreover, by our assumption on $v, u_{\delta}$ is smooth in $\Omega-S$. We want to prove that $u_{\delta}$ converges to a solution of (7.1) as $\delta \rightarrow 0$. Since $u_{\delta}$ is monotone, we may define

$$
u=\lim _{\delta \rightarrow 0} u_{\delta} .
$$

Denote $N:=\{x \in \Omega: u(x)=-\infty\}$. If $N$ has measure zero, then by Lemma 4.3, $u \in S H_{1}(\Omega)$, and by Theorem $6.1, \mu_{1}[u]=v$. To see that $u$ satisfies the boundary condition $u=\varphi$ on $\partial \Omega$, note that $v=f$ is a Lipschitz function near $\partial \Omega$ and recall that Lemma 2.3 holds for functions satisfying $H_{1}[u] \geq f$ (see Remark 2.2). Hence $u_{\delta}$ is locally uniformly bounded and smooth in $\Omega-S$. Therefore the boundary condition $u=\varphi$ is satisfied and so $u$ is a weak solution of (7.1).

It remains to prove that Lebesgue measure $|N|=0$. Suppose to the contrary that

$$
|N| \geq \theta
$$

for some constant $\theta>0$. We claim that there exists a positive constant $\eta>0$ such that

$$
v\left(\Omega_{t}\right) \leq(1-\eta) P_{\Omega_{t}}
$$

for all large $t$, where $\Omega_{t}=\{x \in \Omega: u(x) \leq-t\}$, so that $N=\Omega_{\infty}$. This can be proved by a compactness argument. Indeed, if it is not true, there is a sequence of $\left\{t_{j}\right\}$, $t_{j} \rightarrow t_{\infty} \leq \infty$, such that

$$
v\left(\Omega_{t_{j}}\right) \geq\left(1-2^{-j}\right) P_{\Omega_{t_{j}}} .
$$


Let $\varphi_{j}$ be the characteristic function so that

$$
P_{\Omega_{t_{j}}}=\int_{\mathbb{R}^{n}}\left|D \varphi_{j}\right| .
$$

Since $v\left(\Omega_{t_{j}}\right) \leq v(\Omega)$ is uniformly bounded, $\varphi_{j}$ subconverges in $L^{1}$ to the characteristic function $\varphi$ of $\Omega_{t_{\infty}}$ and

$$
\int_{\mathbb{R}^{n}}|D \varphi| \leq \liminf _{j \rightarrow \infty} \int_{\mathbb{R}^{n}}\left|D \varphi_{j}\right|
$$

Since $\Omega_{t}$ is monotone, we have $v\left(\Omega_{t_{j}}\right) \rightarrow v\left(\Omega_{t_{\infty}}\right)$. Hence

$$
v\left(\Omega_{t_{\infty}}\right) \geq \int_{\mathbb{R}^{n}}|D \varphi|,
$$

which is in contradiction with (7.2). Hence (7.13) holds.

Denote $\Omega_{\delta, t}=\left\{x \in \Omega: u_{\delta}(x) \leq-t\right\}$. Recall that $u_{\delta}$ is monotone. Hence for any $t>0,\left|\Omega_{\delta, t}\right| \geq \theta / 2$ provided $\delta$ is sufficiently small. Let $t$ be fixed and $\delta$ vary. By a compactness argument as above, we also have

$$
v\left(\Omega_{\delta, t}\right) \leq(1-\eta) P_{\Omega_{\delta, t}}
$$

when $\delta$ is sufficiently small.

Let $\delta_{t}>0$ be the sup of all such $\delta$. Note that for any fixed $\delta>0$, if

$$
v\left(\Omega_{\delta, t_{j}}\right) \geq(1-\eta) P_{\Omega_{\delta, t_{j}}}
$$

for a monotone sequence $t_{j} \rightarrow t_{0}$, from (7.14) and (7.15) we have

$$
v\left(\Omega_{\delta, t_{0}}\right) \geq(1-\eta) P_{\Omega_{\delta, t_{0}}} .
$$

Note that for any sequence $t \rightarrow t_{0}$, there is a monotone subsequence of $t$ which converges to $t_{0}$. Hence we have

$$
\liminf _{t \rightarrow t_{0}} \delta_{t} \geq \delta_{t_{0}}
$$

Therefore for any $T>0$, we can choose $\delta>0$ sufficiently small such that (7.16) holds for all $t \in(0, T]$. Now we fix $T=T_{1}$, where $T_{1}$ is given in (3.9). By Step 1 above, $u_{\delta}$ is a bounded function and $u_{\delta} \in \mathrm{BV}(\Omega)$. Hence the proof of Theorem 3.1 is valid (see Remark 3.1) and we obtain

$$
\inf u_{\delta} \geq-C
$$

for some $C>0$ depending on $n,|\Omega|, \inf _{\partial \Omega} u_{\delta}$, and $\eta$, but independent of $\delta$. Sending $\delta \rightarrow 0$, we find that $u$ is bounded from below, a contradiction. Hence $|N|=0$ and $u$ is a weak solution to (7.1).

Remark 7.1. Condition (7.2) corresponds to that in [7-9]. When $v$ (more precisely its density) is a bounded function, (7.2) implies (7.8) for a small $\eta$ [9].

Remark 7.2. A weak solution is usually not $C^{2}$ smooth if $v$ is not Lipschitz continuous. This is easily seen by considering functions of one variable, $u=u\left(x_{1}\right)$. However, if $n \leq 7$ and $v$ is a bounded function and the weak solution is a minimizer of the functional (7.5), then the graph of the solution is a $C^{2, \alpha}$ hypersurface if $v$ is a Hölder continuous function, or $C^{1, \alpha}$ if $v$ is a bounded nonnegative function [17]. 


\section{Remarks}

We include an example showing that some potential-theoretical properties which hold for the $p$-Laplace equation and the $k$-Hessian equation [12, 15, 27-30] may not hold for curvature equations.

Let

$$
u_{c}(x)= \begin{cases}a(r-1)^{\delta} & \text { if } r \geq 1, \\ -b(1-r)^{\theta}-c & \text { if } 0 \leq r<1,\end{cases}
$$

where $r=|x|, a, b>1, c \geq 0, \delta, \theta \in(0,1 / 2)$ are positive constants. Then $H_{1}\left[u_{0}\right]$ is positive and Hölder continuous near $\partial B_{1}$, but $u_{0} \notin C^{1}$, since $\left|D u_{0}\right|=\infty$ on the sphere $\{|x|=1\}$. As remarked at the end of the previous section, the graph of $u_{0}$ is $C^{2, \alpha}$ for some $\alpha>0$.

If $c>0$, then $u_{c}$ is $H_{1}$-subharmonic, and can be approximated by smooth $H_{1}$ subharmonic functions. Therefore weak solutions to the Dirichlet problem (7.1), without the restriction (7.2), are not unique in general. We note that the corresponding uniqueness problem for the $p$-Laplace equation and the $k$-Hessian equations remains open.

When $c>0$, we also see that the Wolff potential estimate (see, e.g., [15, 30]) does not hold for the mean curvature equation, and an $H_{1}$-subharmonic function may not be quasi-continuous, as the capacity of $\partial B_{1}$ is positive.

Acknowledgments. The authors would like to thank Luigi Ambrosio for reading the paper carefully and for his comments which clarified and greatly improved the presentation of the paper.

This work was supported by ARC grants DP0664517 and DP0879422; and NSFC grants 10428103, 10671064 and 10971061.

\section{References}

[1] Ambrosio, L., Soner, H. M.: Level set approach to mean curvature flow in arbitrary codimension. J. Differential Geom. 43, 693-737 (1996) Z Zbl 0868.35046 MR 1412682

[2] Dai, Q. Y., Trudinger, N. S., Wang, X.-J.: The mean curvature measure. arXiv:0912.0341(2009)

[3] Finn, R., Lu, J.: Regularity properties of H-graphs. Comment. Math. Helv. 73, 379-399 (1998) Zbl 0912.53006 MR 1633355

[4] Federer, H.: Curvature measures. Trans. Amer. Math. Soc. 93, 418-491 (1959) Zbl 0089.38402 MR 0110078

[5] Federer, H.: Geometric Measure Theory. Springer, New York (1969) Zbl 0176.00801 MR 0257325

[6] Gilbarg, D., Trudinger, N. S.: Elliptic Partial Differential Equations of Second Order. 2nd ed., Springer (1983) Zbl 0562.35001 MR 0737190

[7] Giaquinta, M.: On the Dirichlet problem for surfaces of prescribed mean curvature. Manuscripta Math. 12, 73-86 (1974) Zbl 0276.35038 MR 0336532

[8] Giusti, E.: Boundary value problems for non-parametric surfaces of prescribed mean curvature. Ann. Scuola Norm. Sup. Pisa 4, 501-548 (1976) Zbl 0344.35036 MR 0482506

[9] Giusti, E.: On the equation of surfaces of prescribed mean curvature. Existence and uniqueness without boundary conditions. Invent. Math. 46, 111-137 (1978) Zbl 0381.35035 MR 0487722 
[10] Giusti, E.: Generalized solutions for the mean curvature equation. Pacific J. Math. 88, 297321 (1980) Zbl 0461.49024 MR 0607982

[11] Giusti, E.: Minimal Surfaces and Functions of Bounded Variations. Birkhäuser, Boston (1984) Zbl 0545.49018 MR 0775682

[12] Heinonen, J., Kilpelainen, T., Martio, O.: Nonlinear Potential Theory of Degenerate Elliptic Equations. Oxford Univ. Press (1993) Zbl 0780.31001 MR 1207810

[13] Korevaar, N.: An easy proof of the interior gradient bound for solutions to the prescribed mean curvature equation. In: Nonlinear Functional Analysis and its Applications, Proc. Sympos. Pure Math. 45 (Part 2), Amer. Math. Soc., 81-89 (1986) Zbl 0599.35046 MR 0843597

[14] Korevaar, N.: A priori interior gradient bounds for solutions to elliptic Weingarten equations. Ann. Inst. H. Poincaré Anal. Non Linéaire 4, 405-421 (1987) Zbl 0644.35041 MR 0921546

[15] Labutin, D. A.: Potential estimates for a class of fully nonlinear elliptic equations. Duke Math. J. 111, 1-49 (2002) Zbl 1100.35036 MR 1876440

[16] Liang, F. T.: Harnack's inequalities for solutions to the mean curvature equation and to the capillarity problem. Rend. Sem. Mat. Univ. Padova 110, 57-96 (2003) Zbl 1121.35056 MR 2033001

[17] Massari, U.: Esistenza e regolarità delle ipersuperfice di curvatura media assegnata in $R^{n}$. Arch. Ration. Mech. Anal. 55, 357-382 (1974) Zbl 0305.49047 MR 0355766

[18] Miranda, M.: Superficie minimale illimitate. Ann. Scuola Norm. Sup. Pisa Cl. Sci. (4) 4, 313 322 (1977) Zbl 0352.49020 MR 0500423

[19] Pucci, P., Serrin, J.: The Harnack inequality in $R^{2}$ for quasilinear elliptic equations. J. Anal. Math. 85, 307-321 (2001) Zbl 1011.35055 MR 1869613

[20] Pucci, P., Serrin, J.: The strong maximum principle revisited. J. Differential Equations 196, 1-66 (2004) Zbl 1109.35022 MR 2100819

[21] Santaló, L. A.: Integral Geometry and Geometric Probability. Addison-Wesley (1976) Zbl 0342.53049 MR 0433364

[22] Schneider, R.: Convex Bodies: the Brunn-Minkowski Theory. Cambridge Univ. Press, Cambridge (1993) Zbl 1143.52002 MR 1216521

[23] Trudinger, N. S.: On Harnack inequalities and their application to quasilinear elliptic equations. Comm. Pure Appl. Math. 20, 721-747 (1967) Zbl 0153.42703 MR 0226198

[24] Trudinger, N. S.: On the analyticity of generalized minimal surfaces. Bull. Austral. Math. Soc. 5, 315-320 (1971) Zbl 0231.35014 MR 0301343

[25] Trudinger, N. S.: Harnack inequalities for nonuniformly elliptic divergence structure equations. Invent. Math. 64, 517-531 (1981) Zbl 0501.35012 MR 0632988

[26] Trudinger, N. S.: A priori bounds and necessary conditions for solvability of prescribed curvature equations. Manuscripta Math. 67, 99-112 (1990) Zbl 0703.35070 MR 1037998

[27] Trudinger, N. S., Wang, X.-J.: Hessian measures I. Topol. Methods Nonlinear Anal. 10, 225239 (1997) Zbl 0915.35039 MR 1634570

[28] Trudinger, N. S., Wang, X.-J.: Hessian measures II. Ann. of Math. 150, 579-604 (1999) Zbl 0947.35055 MR 1726702

[29] Trudinger, N. S., Wang, X.-J.: Hessian measures III. J. Funct. Anal. 193, 1-23 (2002) Zbl 1119.35325 MR 1923626

[30] Trudinger, N. S., Wang, X.-J.: On the weak continuity of elliptic operators and applications to potential theory. Amer. J. Math. 124, 369-410 (2002) Zbl 1067.35023 MR 1890997

[31] Wang, X.-J.: Interior gradient estimates for mean curvature equations. Math. Z. 228, 73-81 (1998) Zbl 0909.35022 MR 1617971 